\title{
Textile Carbon-Flächenelektroden für elektrochemische Applikationen in der Bauindustrie
}

\author{
Kristina Ahlborn ${ }^{1}$, Frank Gerlach ${ }^{1}$, Birgit Wolf ${ }^{2}$, Kirstin Hoffmann ${ }^{2}$ und Winfried Vonau ${ }^{1}$ \\ ${ }^{1}$ Kurt-Schwabe-Institut für Mess- und Sensortechnik e.V. Meinsberg, Waldheim, Deutschland \\ ${ }^{2}$ Institut für Textil-und Ledertechnik (ITL), Reichenbach, Deutschland \\ Kontakt:ahlborn@ksi-meinsberg.de
}

\section{Zusammenfassung:}

Es wird die Entwicklung und Herstellung von neuartigen, textilen Flächenelektroden auf der Basis von Carbonfasern und /oder carbonhaltigen Beschichtungen vorgestellt. Ziel ist es, derartige Elektrodensysteme zur elektrophysikalischen Entfeuchtung und Entsalzung in der Bauindustrie einzusetzen und dabei die bereits angewandten Technologien durch den Einsatz der neuen, deutlich stabileren Elektrodensysteme effizienter zu machen. Mittels elektrischer und elektrochemischer Methoden, wie z. B. Impedanzspektroskopie und Voltammetrie werden Carbonrovings und die daraus hergestellten, neuentwickelten Flächenelektroden umfassend charakterisiert.

Schlagwörter: textile Carbon-Flächenelektroden, elektrochemische Impedanzspektroskopie, potentiodynamische Methoden, Polarisationswiderstand, Bauwerksentfeuchtung und -entsalzung

\section{Einleitung}

Textile Carbonfasern werden gegenwärtig immer häufiger als Hochleistungsverbundwerkstoffe genutzt und finden in vielen Industriezweigen Anwendung, wie z.B. der Luftfahrtindustrie, dem Maschinenbau, der Automobilindustrie, dem Schiffbau, der Medizintechnik und der Sportartikelindustrie [1]. Dabei können sie einzeln oder auch im Verbund mit Begleitmaterialien, wie z.B. Glas-, Polyamid- oder Aramidfasern eingebracht werden.

Für die Bauindustrie werden auf Carbonfasern basierende Fassadendämmsysteme angeboten. Kommerzielle Armierungsgewebe dienen vorwiegend dem Ziel, die mechanische Festigkeit eines Bauwerks zu verbessern.

Dagegen ist deren Einsatz als großflächiges Elektrodenmaterial, z. B. für elektrophysikalische Entfeuchtungs- und Entsalzungsprozesse in der Bauindustrie, bisher nicht publiziert.

Eindringende Feuchte kann bei Neu- als auch bei Altbaubeständen nicht nur Gebäudeschäden verursachen, sondern infolge Schimmelbildung auch zu gesundheitlichen Risiken führen [2]. Dies erfordert oft zeit- und kostenintensive Sanierungsmaßnahmen. Dabei ist es unabdingbar, eine Analyse des jeweils aktuellen Mauerwerkszustandes qualifiziert durchzuführen.

Flächenelektroden werden in der Bauindustrie in Form von Edelstahlgittern eingesetzt $[3,4]$ und unterliegen prozessbedingt einer besonders hohen elektrochemischen Belastung. Eine frühzeitig einsetzende Korrosion, partiell instabiles Verhalten und die daraus resultierende begrenzte Lebensdauer sind die Folge. Hinsichtlich der Materialeigenschaften wirken Edelstahlelektroden nicht vollständig inert und können ungewollte Reaktionen eingehen, die den Prozessablauf negativ beeinflussen.

In den nachfolgend vorgestellten Forschungssarbeiten werden Möglichkeiten aufgezeigt, dem klassischen Bauarmierungsgewebe die Zusatzfunktion einer elektrochemisch wirksamen Flächenelektrode zu verleihen, ohne dass dabei die bewährten mechanischen Eigenschaften darunter leiden. Mittels potentiodynamischer und impedanzspektroskopischer Methoden werden die elektrochemischen Eigenschaften der textilen Carbon-Elektroden applikationsspezifisch untersucht mit dem Ziel, vorhandene Technologien zur Entfeuchtung und Entsalzung von Mauerwerk [5] durch deren Einsatz wesentlich effizienter zu machen.

\section{Methoden und Materialien}

\section{Materialauswahl Carbonfasern}

Um aus den klassischen Bauarmierungsgeweben elektrochemisch wirksame Flächenelektroden herzustellen, werden in diese Carnbonfaserrovings eingearbeitet, oder sie werden mit einer carbonhaltigen Beschichtungsdispersion behandelt. Die Produktpalette der am Markt erhältlichen Carbon- 
fasern ist sehr groß; sie sind unverarbeitet in Form von Rovings aber auch verarbeitet zu Bändern, Geweben und Gestricken verfügbar. Die Auswahl der Rovings zur Herstellung der Flächenelektroden erfolgte unter Berücksichtigung der für den Applikationszweck erforderlichen textilphysikalischen Anforderungen.

\section{Parameter wie}

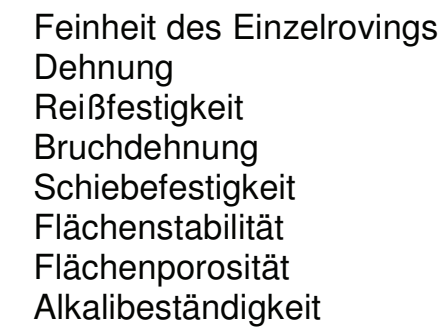

müssen dabei beachtet werden.

\section{Herstellung der Carbon-Flächenelektroden}

Für die Herstellung der Carbon-Flächenelektroden wurden herkömmliche Glasgitter-Armierungsgewebe (Fa. Asglatex) mit kommerziell verfügbaren Carbon-haltigen Latex-Dispersionen beschichtet. Zur Variation des Carbon-Anteils in der Beschichtung wurden die Carbon-haltigen Latex-Dispersionen mit reiner Latexlösung in verschiedenen Mischungsverhältnissen verdünnt.

Bestimmung der elektrischen Leitfähigkeit der Carbonrovings

Eine neu entwickelte Vierelektroden-Anordnung mit RCL-Messbrücke PM6306 der Fa. Fluke diente zur Bestimmung des elektrischen Widerstandes. Stromführende und spannungsabgreifende Kontakte sind bei dieser Anordnung vorteilhaft getrennt, so dass keine störenden Spannungsabfälle an den potentialbildenden Elektroden die Messungen fehlerhaft beeinflussen könnten.

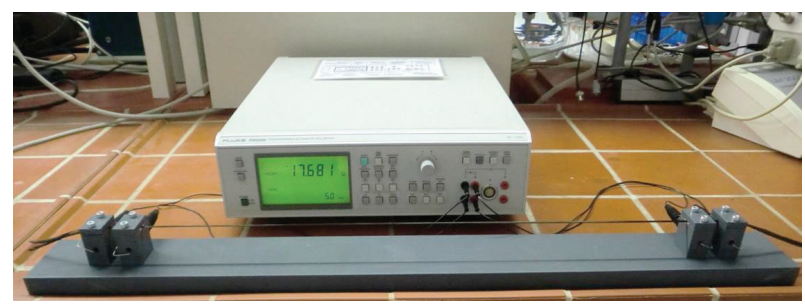

Abb. 1: Widerstandsbestimmung mittels RCL-Messbrücke $(\mathrm{U}=50 \mathrm{mV}, \mathrm{f}=50 \mathrm{~Hz}, 1 \mathrm{kHz}$ bzw. $5 \mathrm{kHz})$ an einem Carbonroving

Zur Vermessung werden die Carbonrovings gespannt und mittels isolierenden Platten fixiert. Die Kontaktabnahme erfolgt über Platinbleche.
Bestimmung der elektrischen Widerstände der Flächenelektroden

Zur Bestimmung der Widerstände der Flächenelektroden wurde die Messanordnung an die Größe der Versuchsmuster angepasst und entsprechend verifiziert.

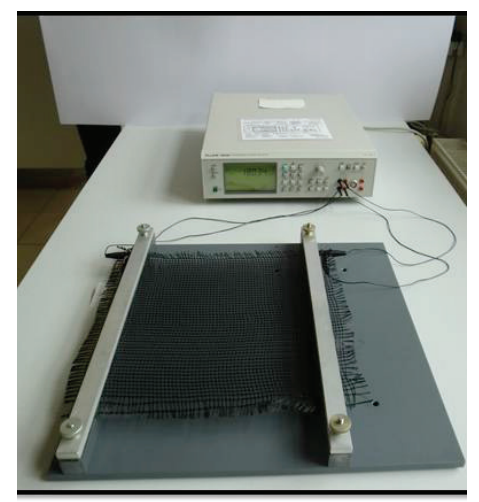

Abb. 2: Widerstandsbestimmung mittels RCLMessbrücke $(\mathrm{U}=50 \mathrm{mV}, \mathrm{f}=50 \mathrm{~Hz}, 1 \mathrm{kHz}$ bzw. $5 \mathrm{kHz})$ an einer Carbon-Flächenelektrode

\section{Elektrochemische Untersuchungen an Carbonrovings}

Für die elektrochemische Charakterisierung wurden die Carbonrovings in Probekörpern hermetisch dicht eingegossen und mit Kontaktelementen versehen (Abb. 3). Derartig konfektioniert garantieren sie reproduzierbare Messbedingungen bei den nachfolgenden Untersuchungen in einer Modelll̈sung.

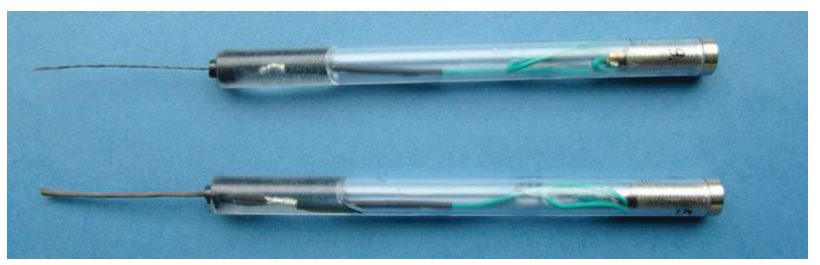

Abb 3: Hermetisch dicht armierte Probekörper mit Carbonrovings

Diese wurden in einer Dreielektrodenanordnung, artähnlich wie bei klassischen Korrosionsmesszellen, durchgeführt. Der Messaufbau ist in Abb. 4 dargestellt.

Neben den Carbonrovings als Arbeitselektrode kam eine Platin-Gegenelektrode und eine mit $\mathrm{KCl}$ gesättigte $\mathrm{Ag} / \mathrm{AgCl}-$ Referenzelektrode zum Einsatz. Zur Datenerfassung wurde das Gamry Reference 600-Messsystem, Fa. Gamry Instruments, genutzt. Die Bestimmung des Ruhepotenzials und des Impedanzspektrums erfolgte in einer $0,1 \mathrm{M}$ $\mathrm{KCl}$-Lösung. Diese Vorgehensweise gewährleistet 
- trotz abweichendem Messmedium zum späteren Einsatz in Baukörpern - eine gute Vergleichbarkeit der unterschiedlichen Elektrodenmaterialien unter Laborbedingungen. Screening und Beurteilung künftiger Elektrodeneigenschaften ist somit möglich. So können Materialleitfähigkeiten, Medieneigenschaften und Interfaceeigenschaften detektiert und verifiziert werden.

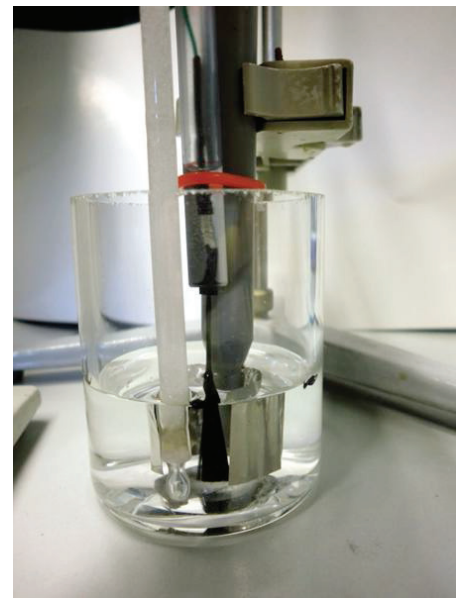

Abb 4: Drei-Elektroden-Messzelle Arbeitselektrode: Carbonroving Gegenelektrode: Platin Referenzelektrode: $\mathrm{Ag} / \mathrm{AgCl} / \mathrm{Cl}^{-}$(ges.) Messmedium: 0,1 M KCl

Elektrochemische Untersuchungen an CarbonFlächenelektroden

Die Abbildung 5 zeigt die Versuchsanordnung zur elektrochemischen Charakterisierung der Flächenelektroden in $0.1 \mathrm{M} \mathrm{KCl}$ als Messlösung.

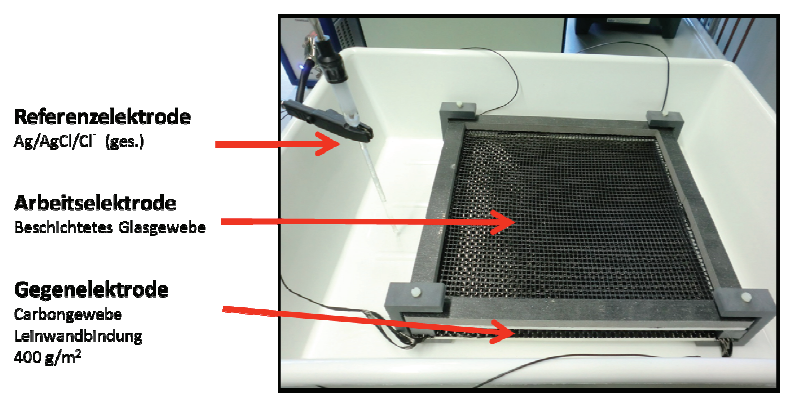

Abb. 5: Versuchsanordnung zur elektrochemischen Charakterisierung der Flächenelektroden in $0.1 \mathrm{M} \mathrm{KCl}$.

Mit den in Abb. 4 und 5 gezeigten Versuchsanordnungen wurden in Kombination mit einem Gamry Reference 600-Messsystem, Fa. Gamry Instruments, potentiodynamische und impedanzspektroskopische Messungen durchgeführt und dabei die elektrochemischen Eigenschaften wie Polarisierbarkeit und Langzeitstabilität der Carbon-Elektroden ermittelt.

\section{Ergebnisse und Diskussion}

Elektrische Widerstände von Carbonrovings und Flächenelektroden

Eine Übersicht zu den ermittelten elektrischen Widerständen an Carbonfasern verschiedener Hersteller zeigt Tabelle 1. In Tabelle 2 sind die elektrischen Widerstände der Flächenelektroden zusammengefasst.

Tab. 1: Elektrische Widerstände der untersuchten Carbonrovings pro $1 \mathrm{~m}$ Länge bei einer angelegten Spannung von $50 \mathrm{mV}$

\begin{tabular}{|l|l|c|}
\hline Muster & Name & $\begin{array}{c}\text { Widerstand } \\
\text { in } \boldsymbol{\Omega}\end{array}$ \\
\hline & & bei $1 \mathrm{kHz}$ \\
\hline $\mathbf{1}$ & NF-24 & 35,34 \\
\hline $\mathbf{2}$ & UMS-12000 & 44,28 \\
\hline $\mathbf{3}$ & IMS-12000 & 63,6 \\
\hline $\mathbf{4}$ & NF-6 & 69,9 \\
\hline $\mathbf{5}$ & NF-12 & 34,96 \\
\hline $\mathbf{6}$ & UMS40 F23 & 23,28 \\
\hline $\mathbf{7}$ & HTS5631 & 33,4 \\
\hline $\mathbf{8}$ & HTA5131 & 70,88 \\
\hline $\mathbf{9}$ & SIGRAFIL C & 460,2 \\
\hline
\end{tabular}

Tab. 2: Elektrische Widerstände der untersuchten Flächenelektroden bei einer angelegten Spannung von 50 $\mathrm{mV}$ und $25 \mathrm{~cm}$ Länge

\begin{tabular}{|l|l|c|}
\hline Muster & Beschichtung & $\begin{array}{c}\text { Widerstand } \\
\text { in } \boldsymbol{\Omega}\end{array}$ \\
\hline & & bei $1 \mathrm{kHz}$ \\
\hline V001 & 20\% Dispersion & n.b. \\
\hline V002 & $35 \%$ Dispersion & $20 \mathrm{M}$ \\
\hline V003 & 50\% Dispersion & $18 \mathrm{k}$ \\
\hline V004 & $\begin{array}{l}\text { Latex 50\% } \\
\text { Dispersion 100\% }\end{array}$ & $1,21 \mathrm{k}$ \\
\hline V005 & Dispersion 100\% & $1,89 \mathrm{k}$ \\
\hline V006 & 20\% Dispersion & n.b. \\
\hline V007 & 35\% Dispersion & $700 \mathrm{k}$ \\
\hline V008 & 50\% Dispersion & $18,1 \mathrm{k}$ \\
\hline V009 & $\begin{array}{l}\text { Latex 50\% } \\
\text { Dispersion } 100 \%\end{array}$ & $3,82 \mathrm{k}$ \\
\hline V010 & Dispersion 100\% & $1,66 \mathrm{k}$ \\
\hline
\end{tabular}

n.b. nicht bestimmbar

\section{Charakterisierung der Carbonrovings}

Die Ergebnisse der in Abbildung 6 dargestellten Impedanzmessungen lassen darauf schließen, dass alle untersuchten Carbonrovings keine nennenswerten Deckschichten aufweisen und bei höheren Frequenzen ab $1 \mathrm{kHz}$ nahezu Ohmsches Verhalten zeigen. 
Die modellhaft abgeleiteten Polarisationswiderstände bei sehr kleinen Anregungsfrequenzen sind auf Grund der Filamentstruktur der Carbonrovings mit 7 bis $9 \mu \mathrm{m}$ Durchmesser der Einzelfilamente und der daraus resultierenden hohen spezifischen Oberfläche wesentlich kleiner als bei massiven Elektrodenkörpern.

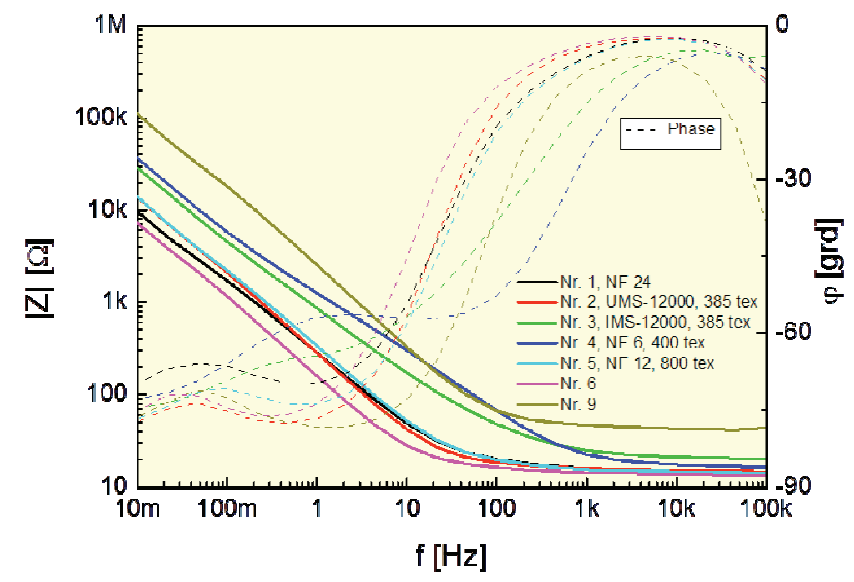

Abb. 6: Impedanzspektren in 0,1 M Kaliumchloridlösung (Arbeitselektrode: Carbonroving, Gegenelektrode: Platinring, Bezugselektrode: $\mathrm{Ag} / \mathrm{AgCl} / \mathrm{Cl}^{-}$(ges.), Amplitude $10 \mathrm{mV}$ )

Auf Grundlage dieser Elektrodeneigenschaften eignen sich die Rovings für textile Flächenelektroden sowohl als Aktorelemente zur aktiven Entfeuchtung und Entsalzung von Baukörpern als auch zur sensorischen Detektion der Feuchte und der Leitfähigkeit. Diese Aussage sollte immaus77 Zuge der Projektarbeit durch andere Untersuchungsmethoden überprüft werden Hierzu eignen sich sowohl die zyklische Voltammetrie als auch die Chronoamperometrie.

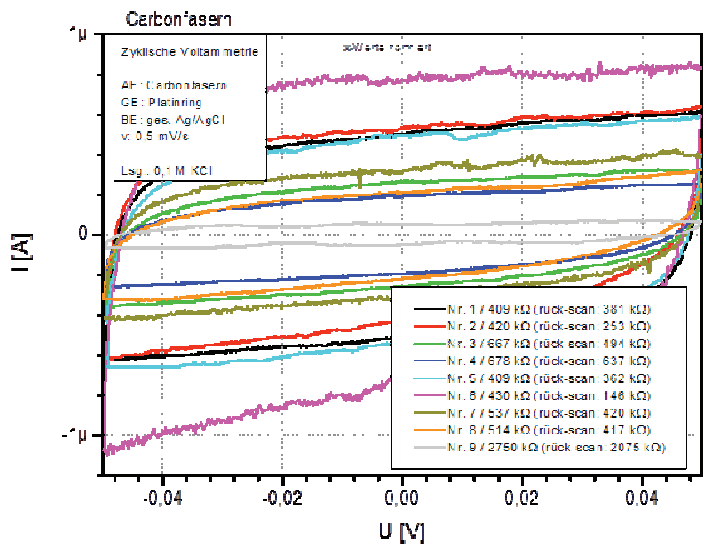

Abb. 7: Zyklische Voltammogramme (normiert) der untersuchten Carbonrovings bei einer angelegten Spannung von $-50 \mathrm{mV}$ bis $+50 \mathrm{mV}$ und einer Scangeschwindigkeit von $0,5 \mathrm{mV} / \mathrm{s}$ (GE: Platinring, $\mathrm{BE}: \mathrm{Ag} / \mathrm{AgCl} / \mathrm{Cl}^{-}$ges.)
Beide Verfahren wurden zusätzlich zur Charakterisierung der 9 Testrovings angewendet. Die zyklische Voltammetrie ermöglicht es, durch Messungen in einem begrenzten Spannungsbereich um das Ruhepotenzial ebenfalls Polarisationswiderstände aus den Kurven zu ermitteln. Dabei wurden in der Darstellung in Abb. 7 die Ruhepotenziale auf $0 \mathrm{~V}$ normiert und ein Spannungsbereich von +/- 50 $\mathrm{mV}$ realisiert. Damit sind die Störungen des Gesamtsystems gering und es kann aus den zu ermittelnden Steilheiten ebenfalls auf den Polarisationswiderstand geschlossen werden.

Einen ähnlichen Informationsgehalt bieten Messungen mittels Chronoamperometrie (Abb. 8). Hierbei wurde das System ebenfalls jeweils $5 \mathrm{~s}$ mit +/- $50 \mathrm{mV}$ beaufschlagt und aus dem nach der Impulszeit ermittelten Messwert der Polarisationswiderstand errechnet.

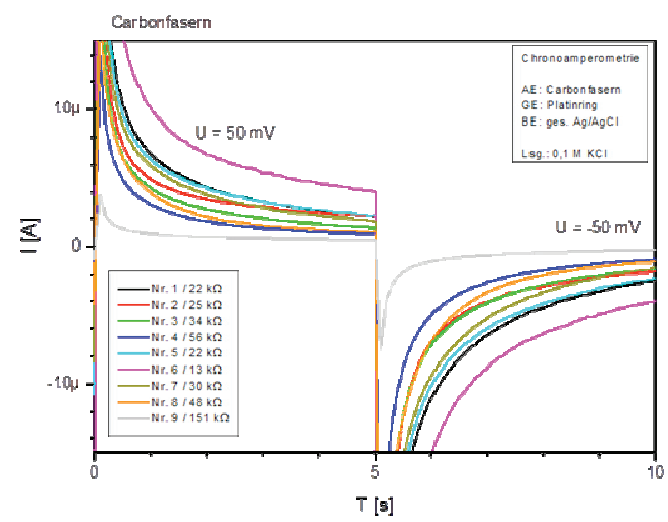

Abb. 8: Chronoamperometrie der untersuchten Carbonrovings bei einer angelegten Spannung von +/$50 \mathrm{mV}$ und einer Impulszeit von jeweils $5 \mathrm{~s}$ (GE: Platinring, $\mathrm{BE}: \mathrm{Ag} / \mathrm{AgCl} / \mathrm{Cl}^{-}$(ges.)

Vergleicht man die Ergebnisse aus den drei Messverfahren (Abb. 9), so kann eine eindeutige Zuordnung der Reihenfolge der Messwerte zu dem entsprechenden Roving getroffen werden, obwohl die Absolutwerte nicht exakt übereinstimmen. Die größten Abweichungen liefert hierbei das Verfahren der Zyklischen Voltammetrie, wobei bei diesem Verfahren die Absolutwerte ganz erheblich von der Scangeschwindigkeit abhängig sind. Damit eignen sich alle drei Messverfahren für Materialscreenings zur Auswahl geeigneter Rovingmaterialien.

Für den praktischen Einsatz kann somit ein geeignetes Messverfahren zur Charakterisierung und Prozessbegleitung favorisiert werden.

Auch die Flächengewebe wurden mit den drei beschriebenen elektrochemischen Messmethoden Impedanzspektroskopie, Zyklische Voltammetrie und Chronoamperometrie charakterisiert. Neben einem unbeschichtetem Carbongewebe in Lein- 
wandbindung mit einem Flächengewicht von $400 \mathrm{~g} / \mathrm{m}^{2}$ wurden verschiedene carbonbeschichtete Glasfasergewebe getestet.

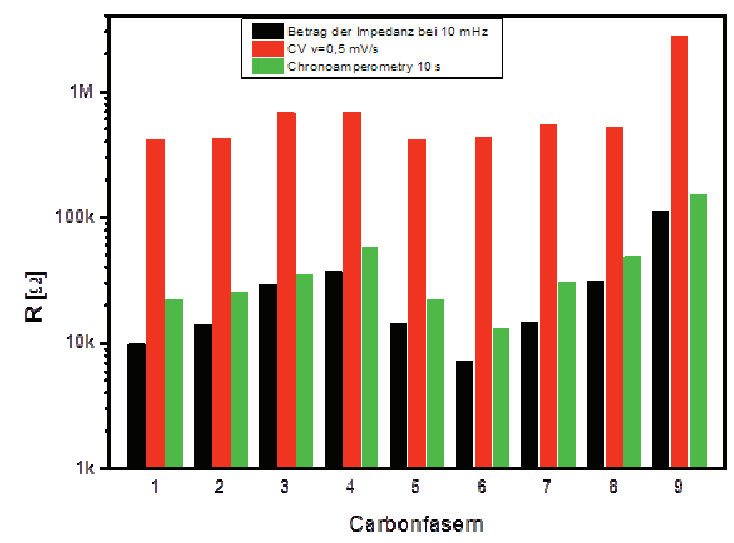

Abb. 9: Vergleich der ermittelten Polarisationswiderstände mittels Impedanzspektroskopie, Zyklischer Voltammetrie und Chronoamperometrie an den Carbonrovings

Die Ergebnisse dieser Untersuchungen zeigt Abbildung $10 \mathrm{im}$ Vergleich zu einer unbeschichteten Carbonfaser. Dabei unterscheiden sich die Gewebe in der Art der Carbonbeschichtung, insbesondere in der Schichtdicke und dem Carbongehalt der Beschichtungslösung.

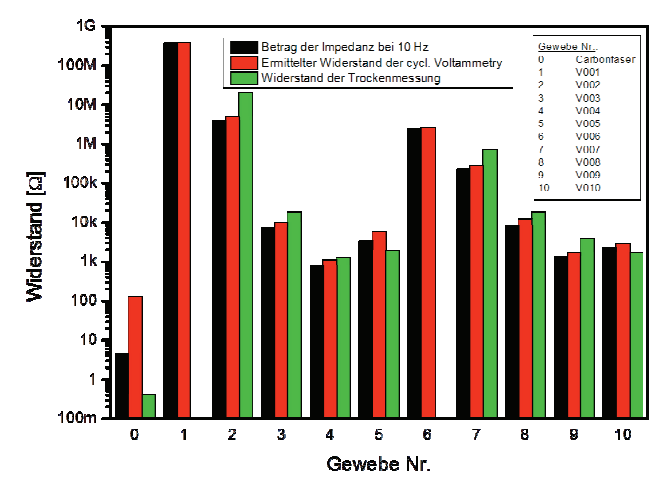

Abb. 10: Vergleich der ermittelten Polarisationswiderstände mittels Impedanzspektroskopie, Zyklischer Voltammetrie und Chronoamperometrie an den Carbonflächenelektroden

\section{Zusammenfassung}

Im Rahmen des geförderten Projektes werden reine Carbongewebe gemeinsam mit mittelständischen Unternehmen und dem Institut für Textil-und Ledertechnik (Reichenbach) in unterschiedlichen
Herstellungstechnologien entwickelt und gefertigt. Diese Gewebe ermöglichen eine hohe Langzeitstabilität und elektrochemische Beständigkeit. Die carbonhaltigen Beschichtungen verbessern das Handling, indem die Stabilität und die Verzugsfestigkeit des Gewebes zunimmt, ohne die hervorragenden elektrochemischen Eigenschaften der Flächenelektroden zu beeinträchtigen. Dies eröffnet eine Vielzahl von Anwendungen, insbesondere in der Bauwirtschaft und Bausanierung.

Die Kombination des etablierten elektroosmotischen Entfeuchtungsverfahrens [6] mit dem Einsatz neuentwickelter, carbonhaltiger Flächenelektroden stellt eine innovative Neuerung dar, welche die bekannten Nachteile der Verfahren des Trockenlegens und der Entsalzung von Baukörpern in effizienter Weise eliminiert.

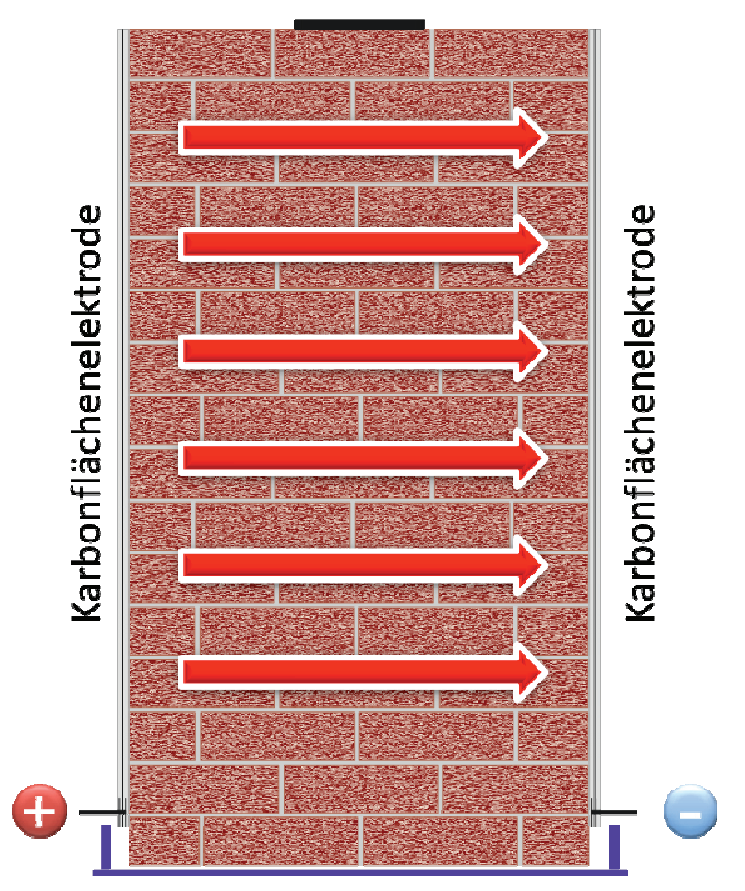

Abb. 11: Schematische Darstellung zum Einsatz der Carbon-Flächenelektroden bei der Entfeuchtung von Mauerwerk.

Die Flächenelektroden ermöglichen, wie in der Prinzipdarstellung des realisierten Modellmauerwerks in Abb. 11 dargestellt, einen homogenen Feldlinienverlauf, niedrige Polarisationswiderstände an den Elektroden und auf Grund der Materialeigenschaften sehr hohe Lebensdauern, ohne eine Verringerung der Effizienz des Verfahrens. 


\section{Literatur}

[1] WOHLMANN, B.: Kohlenstoff-Fasern. In AKV (Hrsg.) „Handbuch Faserverbund -„Grundlagen, Verarbeitung, Anwendungen“, Vieweg+Teubner/GWV Fachverlage $\mathrm{GmbH}$, Wiesbaden, 2010

[2] WEBER, J.; HAFKESBRINK, V.(Hrsg.): Bauwerksabdichtung in der Altbausanierung, Vieweg+Teubner Verlag/GWV Fachverlage $\mathrm{GmbH}$, Wiesbaden 2008

[3] DAUBERSCHMIDT, C.; SODEIKAT, C.; SCHIESSL, P.; GEHLEN, C.: Monitoring von Verkehrsbauten - kontinuierliche Zustandserfassung, Tiefbau 3(2008)135-140

[4] SCHNECK, U.; GRÜNZIG, H. (CITec GmbH): Modellhafte elektrochemische Salzminderung von Objekten aus Elbsandstein am Dresdner Zwinger. Abschlußbericht zu Forschungsprojekt Aktenzeichen 23783-45, Deutsche Bundesstiftung Umwelt, November 2008

[5] VONAU, W.; BERTHOLD, F.; AHLBORN, K.; GERLACH, F: Sensors for the monitoring of corrosion risks, corrosion reducing procedures and critical conditions of buildings, especially of reinforced concrete.

IMCS 2014, 15th International Meeting on Chemical Sensors, Buenos Aires, Argentinien, 16.-19.03.2014

[6] BRAUN, F.-J., PÖtZSCHE, P., WAGEMANN, G: Elektroosmotische Bauwerkstrockenlegung, VEB Verlag für Bauwesen, Berlin 1969

\section{Danksagung}

Das Kooperationsvorhaben „Textile Carbon-Flächenelektroden für elektrochemische Applikationen in der Bauindustrie" wurde im Rahmen der ZIM-Förderung aus Haushaltsmitteln des Bundesministeriums für Wirtschaft und Technologie (BMWi) über die Arbeitsgemeinschaft industrieller Forschungsvereinigungen „Otto von Guericke“ e.V. unter dem Förderkennzeichen KF2341620AG4 gefördert. Die Autoren danken für die finanzielle Unterstützung. 